\title{
A Retrospective Study on Evaluation of Patients with Uterine Fibroid in a Tertiary Care Hospital
}

\author{
Anju Mam Thomas, Blessy Rachal Boban, Jiya Ann Mathew
}

\author{
Pharm.D (Doctor of Pharmacy) Interns, Pushpagiri Medical College Hospital, Tiruvalla, Kerala
}

\begin{abstract}
Uterine fibroids are a major cause of morbidity in women of reproductive age. Hence it is important to evaluate the occurrence of fibroid. An observational retrospective study was carried out in Obstetric and Gynecology Department over a period of 2 months. Each of the cases was scrutinized for sociodemographic, clinical profile and other necessary information. In this study, Fibroid was found to be predominant in premenopausal women. .Parity and number of abortions had no much significance with fibroid diagnosed. The primary management of obese patients were found as weight reduction and diet control. Hysterectomy was done based on large fibroid size.
\end{abstract}

\section{KEYWORDS: Fibroid, Hysterectomy, Parity}

\section{INTRODUCTION}

Uterine fibroids are a major cause of morbidity in women of a reproductive age and sometimes even after menopause. There are several factors that are attributed to underlie the development and incidence of these common tumors, but this further corroborates their relatively unknown etiology. The most likely presentation of fibroids is by their effect on the woman's menstrual cycle or pelvic pressure. ${ }^{1}$ Uterine fibroids (also known as leiomyomas or myomas) are the commonest benign uterine tumors, with an estimated incidence of $20 \%-40 \%$ in women during their reproductive years. They are monoclonal tumors of the uterine smooth muscle cells and consist of large amounts of extracellular matrix that contain collagen, fibronectin, and proteoglycan. Even though their pathogenesis is not clearly known, there is considerable evidence that estrogens and progestogens proliferate tumor growth, as the fibroids rarely appear before menarche and regress after menopause. They are classified by their location relative to the layers of the uterus (as subserous, intramural, or submucous) and can be single or multiple. Although the etiology of fibroids remains unknown, the ovarian hormones estrogen and progesterone are hypothesized to enhance fibroid growth ${ }^{3}$. Reported risk factors consistent with the hormonal hypothesis include premenopausal status ${ }^{4}$, younger age at menarche ${ }^{5,6}$, and obesity $^{7}$. Never having been married and higher educational attainment ${ }^{8}$ are also reported to be risk factors. History of infertility, young age at first birth, and current alcohol consumption have been associated with increased risk . Reported protective factors include parity ${ }^{9}$ and oral contraceptive use. The common clinical presentations- were abdominopelvic mass (100\%), menorrhagia (95.7\%), infertility (41.9\%), anemia (32.9\%), dysmenorrhoea (12.5\%), abdominopelvic pain (15.8\%), and urinary pressure symptoms (6.8\%).Abdominal myomectomy $(97.3 \%)$ was the main stay of treatment ${ }^{10}$. During pregnancy, fibroids may be a cause of miscarriage, bleeding, abnormal lie and presentation [11]. It is also postulated that larger fibroids may distort or block the fallopian tubes.$^{12}$ Compared to Caucasians, Negroid women are reported to have a higher incidence of uterine fibroid age for age. The incidence of uterine fibroid in this study (29.3\%) was similar to the $25.9 \%$ reported in Enugu, and higher than the $11 \%$ reported in Caucasian women. The high incidence among
Nigerian women may be associated with race and genetic

factors, which have been implicated as risk factors by several studies which reported higher incidence in black women and women of African descent . 13,14 Typically, fibroids appear as well-defined, solid masses with a whorled appearance. Ultrasonography using the transabdominal and transvaginal routes has been employed most frequently, due to its accessibility and relatively low cost. ${ }^{15}$ Saline infusion sonohysterography-based imaging is usually used as a supplementary or adjunct imaging modality for characterization of focal uterine masses diagnosed on Bmode ultrasound images. Magnetic resonance imaging, while more costly, has been touted as the most sensitive modality for evaluating uterine myomas, particularly for the detection of small fibroids. ${ }^{16}$ Management of a patient with uterine fibroids is highly dependent on the presentation and patient wishes. Other causes of abnormal uterine bleeding, pelvic pressure/pain, and subfertility usually need to be ruled out beforehand. In many cases, the management of the fibroids carries surgical risks, and in some women, the fibroids are best left alone. Women with symptoms who have small fibroids but are close to menopause or who are trying to conceive should be offered conservative treatment with analgesics and hematinics. ${ }^{17}$ Surgical methods are the mainstay of treatment when treatment is necessary. Possible surgical interventions include hysterectomy, or removal of the uterus (and the fibroids with it). Myomectomy is the selective removal of just the fibroids within the uterus. Myomectomy can be done through a hysteroscope, laparoscope or with the standard open incision on the abdominal wall. Some treatments have involved boring holes into the fibroid with laser fibers, freezing probes (cryosurgery), and other destructive techniques that do not actually remove the tissue but try to destroy it in place. Surgery is necessary if there is suspicion of malignancy in any case of a leiomyoma or uterine mass. ${ }^{18}$

Another technique for treating fibroids is known as uterine artery embolization (UAE). This technique uses small beads of a compound called polyvinyl alcohol, which are injected through a catheter into the arteries that feed the fibroid. These beads obstruct the blood supply to the fibroid and starve it of blood and oxygen. While this technique has not 
been in use long enough to evaluate long-term effects of UAE versus surgery, it is known that women undergoing UAE for fibroids have a shorter hospital stay than those having surgery but a greater risk of complications and readmissions to the hospital. Studies are underway to evaluate the longterm outcomes of UAE as opposed to surgical treatment. Uterine artery occlusion (UAO), which involves clamping the involved uterine arteries as opposed to injecting the polyvinyl alcohol beads, is currently under investigation as a potential alternative to UAE.

High-intensity focused ultrasound (HIFU) is a relatively new treatment for fibroids and other abnormalities. It is also known as MRgFUS (MRI-guided focused ultrasound) and FUS (focused ultrasound surgery). HIFU uses an ultrasound transducer with higher energy than those used for diagnostic examinations. The device focuses the sound waves, generating heat to destroy the fibroid. MRI imaging may be used for planning and monitoring of treatment.Non-surgical techniques are usually hormonal in nature and include the use of drugs that turn off the production of estrogen from the ovaries (GnRH analogs). These medications are given for three to six months and induce a hypoestrogenic (low estrogen) state. When successful, they can shrink the fibroids by as much as $50 \%$. Side effects of these drugs are similar to the symptoms of menopause and can include hot flashes, sleep disturbance, vaginal dryness, and mood changes. Bone loss leading to osteoporosis after long-term (6 to $12+$ months) use is one complication. This is generally reversed after the treatment ends. These drugs may also be used as preoperative treatment for large leiomyoma to shrink them in order to make the operation less difficult and reduce surgical risk.

Mifepristone (RU-486) is an antiprogestin drug that can shrink fibroids to an extent comparable to treatment with the GnRH analogs. This drug is also used to terminate early pregnancy. Treatment with mifepristone also reduced the bleeding associated with fibroids, but this treatment can be associated with adverse side effects such as overgrowth (hyperplasia) of the endometrium (uterine lining). Mifepristone is not approved by the US Food and Drug Administration (FDA) for the treatment of uterine leiomyomas, and the required dosages (different from those used for termination of early pregnancy) have not been determined.

Danazol (Danocrine) is an androgenic steroid hormone that has been used to reduce bleeding in women with fibroids, since this drug causes menstruation to cease. However, danazol does not appear to shrink the size of fibroids. Danazol is also associated with significant side effects, including weight gain, muscle cramps, decreased breast size, acne, hirsutism (inappropriate hair growth), oily skin, mood changes, depression, decreased high density lipoprotein (HDL or 'good cholesterol') levels, and increased liver enzyme levels.

The administration of raloxifene (Evista), a drug used to prevent and treat osteoporosis in postmenopausal women, has been shown to decrease the size of fibroids in postmenopausal women, but results with this therapy in premenopausal women have been conflicting.

Low dose formulations of oral contraceptives are also sometimes given to treat the abnormal bleeding associated with fibroids, but these do not shrink the fibroids themselves.

\section{MATERIALS AND METHODS}

This was an observational retrospective study carried out in Obstetric and Gynecology Department. over a period of 2 months. In this study a total of 20 cases with uterine fibroid were enrolled during study period. The medical records of all 20 cases of uterine fibroids managed during the period were retrieved from the hospital medical record department and retrospectively reviewed. Each of cases was scrutinized for socio-demographic, clinical profile and other necessary information. Data were expressed as number (percentage) in tabular and graphical form. Appropriate statistical test was applied. P value $<0.05$ was taken as level of significance and data were entered and analyzed by MS excel-2014.

\section{RESULTS}

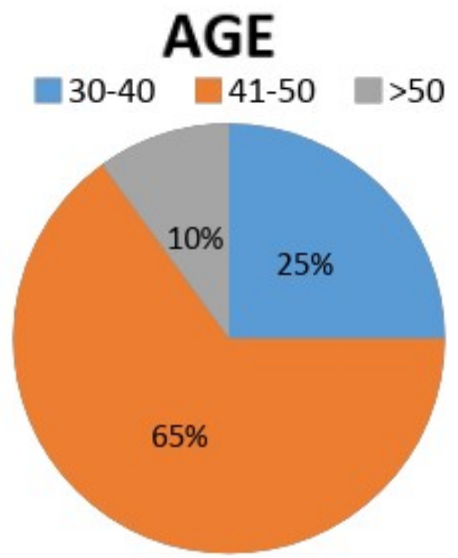

Out of total patients, $65 \%$ of patients belongs to the age group $41-50 ; 25 \%$ of patients belongs to the age group $30-$ 40 and $10 \%$ of patients belongs to the age group greater than 50 .

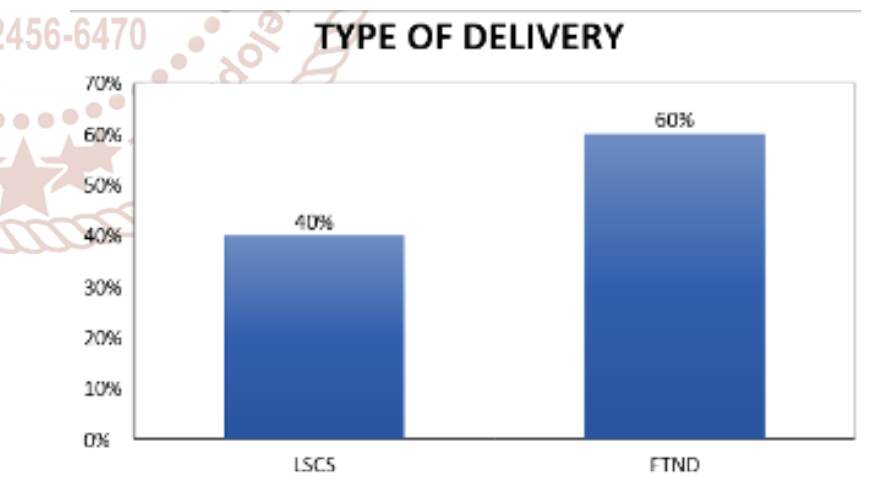

$60 \%$ of the total population has undergone Full Term

Normal Delivery whereas the remaining $40 \%$ has undergone Lower Segment Cesarean Section.

\section{PARITY}

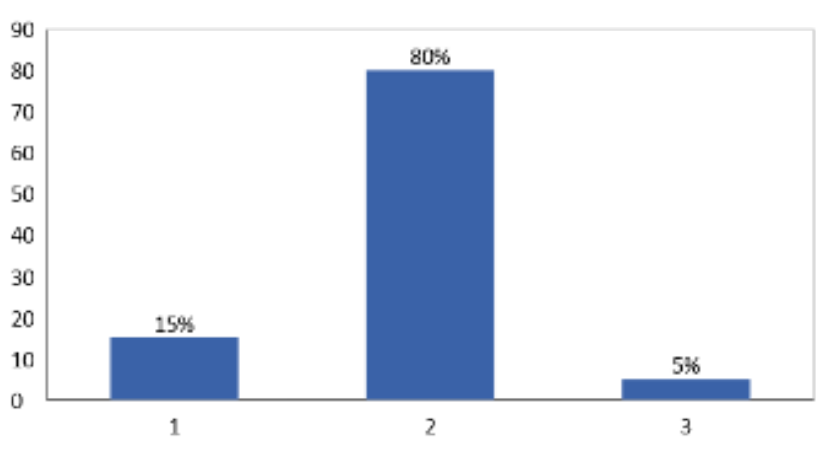


$80 \%$ of total population has parity 2 whereas $15 \%$ has parity 1 and the remaining 5\% has parity 3.No nulliparity has been observed.

\section{STERILIZED}

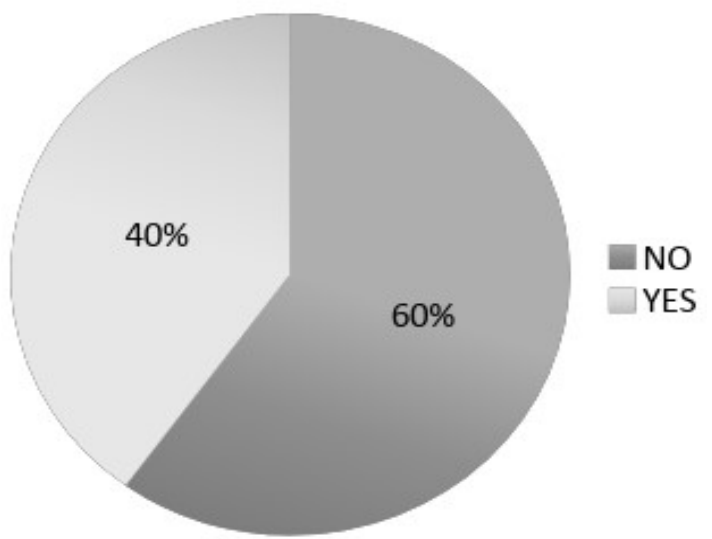

$60 \%$ of total population is not sterilised whereas $40 \%$ has undergone sterilization.

\section{PAST HISTORY}

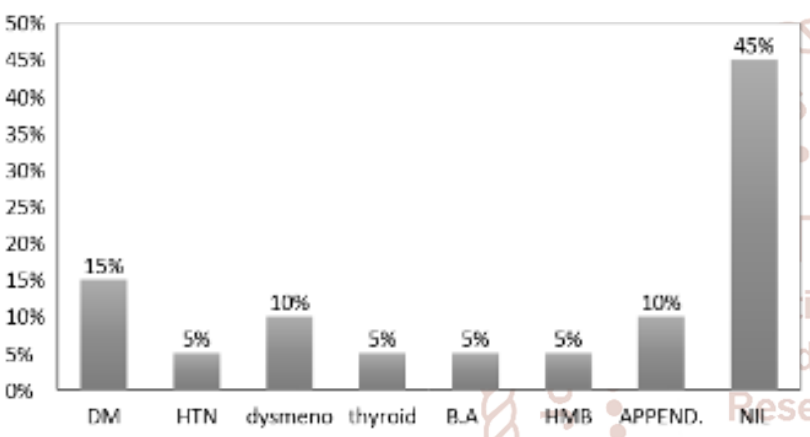

Majority of the population has no past history.15\% had D.M, 10\% had dysmennorhea. Few patients had thyroid, hypertension, bronchial asthma.

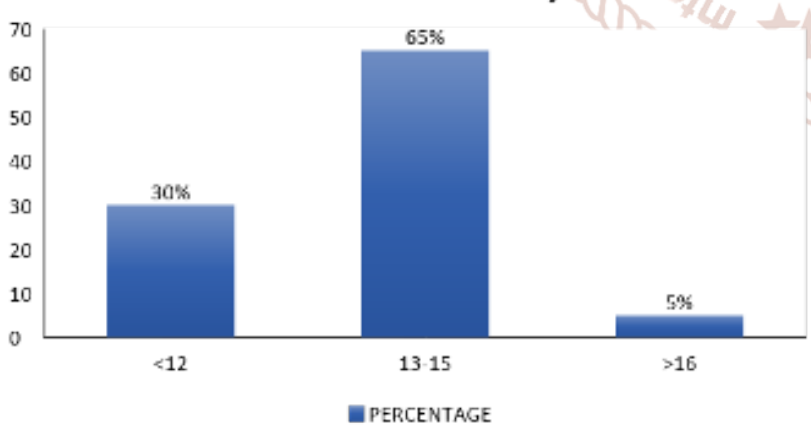

$65 \%$ of total population has menarche within $13-15$ years of age, $30 \%$ has menarche at less than 12 years of age and the remaining $5 \%$ has menarche at age greater than 16 years.

In our study, majority of the patients (50\%) had lower abdominal pain, (35\%) had heavy menstrual bleeding and $25 \%$ had dysmennorhea. Only 3 patients had history of abortion. $45 \%$ of the total patients had multiple fibroids.35\% of patients had bulky uterus. The primary management considered for obese patient was weight reduction.

\begin{tabular}{|c|c|c|}
\hline Weight (kg) & No.of cases & Percentage \\
\hline $50-60$ & 13 & 65 \\
\hline $61-70$ & 5 & 25 \\
\hline $71-80$ & 1 & 5 \\
\hline
\end{tabular}

\begin{tabular}{|c|c|c|}
\hline $81-90$ & 1 & 5 \\
\hline
\end{tabular}

$65 \%$ of the women has weight range within $50-61 \mathrm{~kg}$. only $5 \%$ of the women had overweight of greater than $81 \mathrm{~kg}$.

\begin{tabular}{|c|c|c|}
\hline Treatment & No. of cases & Percentage \\
\hline TAH+BSO & 3 & 15 \\
\hline TAH & 2 & 10 \\
\hline D\&C & 6 & 30 \\
\hline T. Meftal & 4 & 20 \\
\hline Diet & 1 & 5 \\
\hline
\end{tabular}

$30 \%$ of patients were recommended D\&C, $20 \%$ with Tablet Meftal 500mg, 10\% with Total Abdominal Hysterectomy, 15\% with TAH+BSO and remaining 5\% with strict diet control.

\section{CONCLUSION}

Uterine fibroid is a common concern in women causing heavy bleeding and pain symptoms which can have a negative impact on different aspects in women's life.In this study ,majority (65\%) of the women population belongs to $41-50$ age group are found to have fibroid diagnosed. Women having menarche at age greater than 16 years of age was found to have less chance for fibroid. For overweight patients, weight reduction and strict diet control were proposed as the primary management. Majority of the patients had symptoms of heavy menstrual bleeding and low backache $.45 \%$ of the total population had no past history of dysmennorhea etc.Fibroid was predominant in premenopausal women.Parity and number of abortions had no much significance with fibroid diagnosed.

\section{REFERENCE}

[1] Rein MS, Barbieri RL, Friedman AJ. Progesterone: a critical role in the pathogenesis of uterine myomas. Am J Obstet Gynecol. 1995; 172(1 Pt1):14-18.

[2] Andersen J. Growth factors and cytokines in uterine leiomyomas. Semin Reprod Endocrinol. 1996; 14(3): 269-282.

[3] Buttram VC, Reiter RC. Uterine leiomyomata: etiology, symptomatology, and management. 1981, 36; 143-45.

[4] Marshall LM, Spiegelman D, Barbieri RL, et al. Variation in the incidence of uterine leiomyoma among premenopausal women by age and race. Obstet Gynecol 1997; 90:967-73.

[5] Samadi AR, Lee NC, Flanders WD, et al. Risk factors for self-reported uterine fibroids: a case-control study. $\mathrm{Am}$ J Public Healt 1996; 86:858-62

[6] Ross RK, Pike MC, Vessey MP, et al. Risk factors for uterine fibroids: reduced risk associated with oral contraceptives. Br Med J 1986;293:359-63

[7] Michele R Forman, ${ }^{1}$ Lauren D Mangini, ${ }^{1}$ Rosenie Thelus-Jean, ${ }^{2}$ and Mark D Hayward ${ }^{3}$ et al. Life-course origins of the ages at menarche and menopause. Adolesc Health Med Ther. 2013; 4: 1-21

[8] Marshall LM, Spiegelman D, Barbieri RL, et al. Variation in the incidence of uterine leiomyoma among premenopausal women by age and race. Obstet Gynecol 1997;90:967-73.

[9] Parazzini F, Negri E, Vecchia CL, et al. Reproductive factors and risk of uterine fibroids. Epidemiology 1996; 7:440. 
International Journal of Trend in Scientific Research and Development (IJTSRD) @ www.ijtsrd.com eISSN: 2456-6470

[10] Atombosoba. A. Ekine1, Lucky O. Lawani2, Chukwuemeka A. Iyoke3, Israel Jeremiah1, Isa. A. Ibrahim1 Review of the Clinical Presentation of Uterine Fibroid and the Effect of Therapeutic Interventi on Fertility American Journal of Clinical Medicine Research, 20153 (1) . pp 9-13

[11] Vollenhoven BJ, Lawrence AS and Healey DC (1990). Uterine fibroid: a clinical review. British Journal of Obstetrics and Gynaecology 1990: 97: 285-98

[12] Bendifallah, S., J. L. Brun, and H. Fernandez. "[Myomectomy for infertile women: the role of surgery]." J. Gynecol. Obstet. Biol. Reprod. (Paris) 40.8 (2011): 885-901.

[13] Ezugwu EC, Iyoke CA, Ezugwu FO, Ugwu G: Successful pregnancy following myomectomy for giant uterine fibroid in an infertile woman. J Reprod Infertil 2014, 15: 233-236.
[14] Fields KR, Neinstein LS. Uterine myomas in adolescents: case reports and a review of the literature. J Pediatr Adolesc Gynecol. 1996; 9(4):195-198.

[15] Levens ED, Wesley R, Premkumar A, Blocker W, Nieman LK. Magnetic resonance imaging and transvaginal ultrasound for determining fibroid burden: implications for research and clinical care. Am J Obstet Genecol. 2009; 200(5):537.

[16] Wise LA, Palmer JR, Harlow BL, et al. Reproductive factors, hormonal contraception, and risk of uterine leiomyomata in African-American women:a prospective study. Am J Epidemiol. 2004; 159(2):113123.

[17] Simms-Stewart D, Fletcher H. Counselling patients with uterine fibroids: a review of the management and complications. Obstet Gynecol Int. 2012;2012:539365

[18] www.medicinenet.com/uterine_fibroids/article.htm\#u terine_fibroids_definition_and_facts 